\title{
Vehicle Recognition Based on Fourier, Wavelet and Curvelet Transforms - a Comparative Study
}

\author{
Farhad Mohamad Kazemi $^{\dagger}$, Saeed Samadi ${ }^{\dagger+}$, Hamidreza Pourreza ${ }^{\dagger+}$, Mohamad R. Akbarzadeh $^{\dagger++}$ \\ ${ }^{\dagger}$ Islamic Azad University, Department of Artificial Intelligence, Mashhad, Iran \\ ${ }^{+}$Khorasan Science and Technology Park (KSTP), Department of Electronic, Mashhad, Iran \\ ${ }^{\mathrm{HT}}$ Ferdowsi University, Department of Computer Engineering, Mashhad , Iran, \\ ${ }^{\mathrm{HWt}}$ Ferdowsi University, Department of Electrical Engineering, Mashhad, Iran
}

\begin{abstract}
Summary
This paper proposes the application of 3 different kinds of feature extractors to recognize \& classify 5 models of vehicles. These feature extractors are Fast Fourier transform, discrete wavelet transform \& discrete curvelet transform. To justify the correct amount of each feature extractor, we perform each of the mentioned transforms to input images, precisely.

The classifier used in this paper is called k nearest-neighbor. The results of this test show, that the right recognition rate of vehicle's model in this recognition system, at the time of using curvelet transform (Notice, all curvelet coefficients) is $100 \%$.

For decreasing the dimension of feature vectors more $\&$ choosing the best features we've used interclass variance criteria to infraclass variance criteria. As a result of this performance, the size of feature vectors will be extremely decreased. Then, we perform our final impact feature vectors (The best Curvelet coefficients or the best wavelet coefficients or the best Fourier coefficients) to the KNN Classifier. Also, the results of this test show, the right recognition rate of vehicle's model in this recognition system, at the time of using 0.1 of all curvelet coefficients is $100 \%$.The comparison of the 3 proposed approaches for identifying the kind of vehicles showed that curvelet transform can extract better features among the proposed dataset.
\end{abstract}

\section{Introduction}

Recently, vehicle based access control systems for buildings, outdoor sites and even housing estates have became commonplace. Additionally, various traffic monitoring and control systems that depend on user (man+vehicle) identification, such as congestion charging would also benefit by augmenting existing number-plate recognition with an additional authentication mechanism. Given an image containing a backward view of a vehicle (car), a system is proposed here that determines its exact class (model). The aim is to obtain reliable classification of a vehicle in the image from a multitude of possible classes (vehicle types) using a limited number of prior examples.
Although classification of road going vehicles has been a subject of interest in the past, e.g. traffic control systems and toll levy automation, vehicle type recognition has not been considered at this level of accuracy. Instead, Most of the systems either detect (classify vehicle or background) or classify vehicles in broad categories such as cars, buses, heavy goods vehicles (HGVs) etc. [5, 2, 6, 7, 3, 1].

V.S.Petrovic and T.F.Cootes [8] demonstrate that a relatively simple set of features extracted from sections of car's frontal images can be used to obtain good performance verification and recognition of vehicle type. The proposed recognition system in this case is based on recognizing rigid structure samples obtained using specific feature extraction techniques from an image of the object (vehicle). Recognition is initiated through an algorithm that locates a reference segment on the object, in this case the frontal number plate. The location and scale of this segment is used as reference to define a region of interest in the image from which the structure is sampled. A number of feature extraction algorithms that perform this task, including direct and statistical mapping methods are investigated. Feature vectors are finally classified using simple nearest neighbor classification.

Louka Dlagnekov [9] developed an LPR (License Plate Recognition) system for achieving a high recognition rate without needing a high quality video signal from expensive hardware. He also explored the problem of car make and model recognition for purposes of searching surveillance video archives for a partial license plate number combined with some visual description of a car. His proposed methods will provide valuable situational information for law enforcement units in a variety of civil infrastructures.

This paper proposes the application of 3 different kinds of feature extractors to recognize \& classify 5 models of vehicles. To justify the correct amount of each feature extractors, we perform per of the mentioned transforms to 
input images, precisely. The comparison of the 3 proposed approaches for identifying the kind of vehicles showed that curvelet transform can extract better features from the proposed dataset.

We've gathered a data set that includes of 300 images from 5 different classes of vehicles. These 5 classes of vehicles includes of: PEUGEOT 206, PEUGEOT 405, Pride, RENUT5 and Peykan. We've examined 230 pictures as our train data set and 70 pictures as our test data set.

\section{Feature extraction}

\subsection{Fourier Transform}

The representation of the pattern in the frequency domain allows for an easy way of providing different in variances (normalization) to the pattern, such as translation, scale, rotation $[4,10]$.

\subsection{Wavelet Transform}

Wavelet transform belongs to the multiresolution transformation, performing the decomposition of the signal on di1erent (usually diadic) levels [12,13]. We will apply here the discrete wavelet transform, representing the original pattern on many levels, di1ering with the number of details. Applying the wavelets to the pattern representations we have to choose the limited number of levels, representative for the task and normalize their coefficients to provide the appropriate invariances to the translation, scale and rotation [11].

In distinction to the Fourier transform applying the sinusoidal basis functions, the wavelet transformation uses the so-called wavelet basis functions (shortly wavelets) and the scaling functions, both forming the orthogonal or biorthogonal family of basis functions [13,14]. The wavelet functions have good localization abilities in both time and frequency, enabling good representation of the local features of the patterns. From this point of view, the wavelet descriptors are better than Fourier, since they are able to catch small di1erences between patterns.

\subsection{Curvelet transform}

Curvelets as proposed by E. Cand Cand es and D. Donoho [15], constitute a relatively new family of frames that are designed to represent edges and other singularities along signed curves much more efficiently than the traditional wavelet based transforms. For example, as shown in Fig.1(a), it based would take many wavelet coefficients to accurately represent such a curve. Compared with wavelets, curvelets can sent represent a smooth contour with much fewer coefficients for the same precision (Fig.1(b)).

The curvelet transform is a multi-scale transform with frame elements indexed by location, scale and orientation parameters, and have time-frequency localization properties of wavelets but also shows a very high degree of directionality and anisotropy. More precisely, we here use a new tight frame of curvelets recently developed in [16].

Further details about curvelets can be found in $[15,16]$. Fig.2 shows a typical set of curvelet coefficients of the image "PEGEOT 206" divided into 4 scales using the Digital Curvelet Transform (DCT) of [17].

\subsection{Classification}

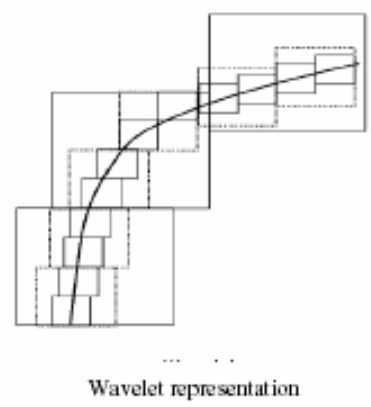

(a)

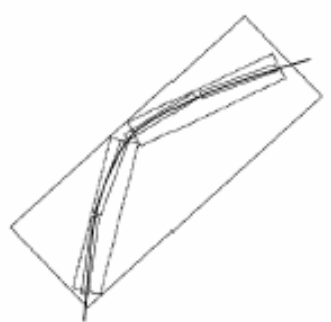

Curvelet representation

(b)
Fig. 1. Comparison of non-linear approximation performance for curvelets and wavelets.

This stage is vehicle classification stage. In this here, the obtained features from stage 2 are used for intelligent classification. The related feature vector in each picture enters to the related classifier.

The $k$ nearest-neighbor is used for classification [19]. The achieved consequences of this algorithm are discussed more in experimental result section. The structure of algorithm for vehicle recognition is shown in Figure. 2. 

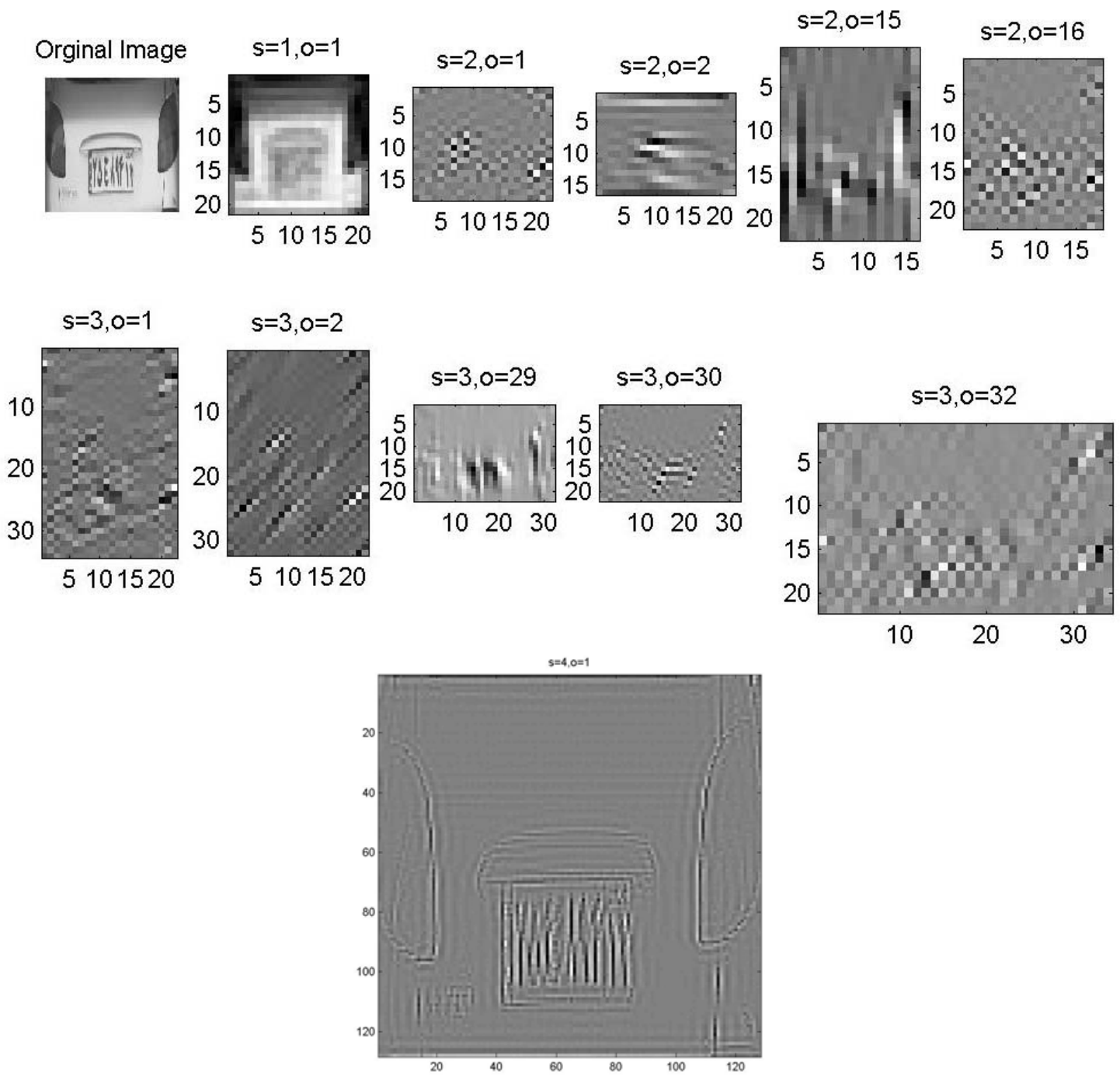

Figure.2. An example of digital curvelet transform of the image "PEGEOT 206". (s: is the scale, o: is the orientation).

As you see in the related picture to scale 4 , orientation 1 the edges have been extracted slowly. 


\section{Experimental results}

For testing the right performance of our proposed algorithm, we've examined 5 common classes of vehicles in Iran, including Peugeot 206, Peugeot 405, Pride, Renault 5 and Peykan. Our total data set used in this study is a set of 300 images from backward view of the mentioned vehicles.

Our training data set include 230 images from backward view of the mentioned vehicles, while our test data set include 70 images from backward view of the mentioned vehicles.

The size of all test \& training images must be normalized to $128 * 128$ pixels.

3.1. The comparative analysis of FFT, Wavelet \& Curvelet descriptors

In this paper, we've proposed a comparative analysis of 3 different transforms, including :

-Fourier transform

-Wavelet transform

-Curvelet transform

The obtained details of performing the achieved coefficients from these transforms are designed when they would be used as feature vector.

To justify the correct amount of obtained features from the transforms, in each recognition algorithm, we've used of all achieved coefficients from it's related transform as feature vector.

\subsubsection{Fourier transform}

As a result of performing Fourier transform to each of input images, 16384 Fourier coefficients were obtained. All of these Fourier coefficients performed to their related classifier as feature vector \& finally our system recognition rate was $97 \%$.

\subsubsection{Wavelet transform}

As a result of performing this transform to each of input images, 16384 wavelet coefficients were obtained. All of these wavelet coefficients (approximation+ detail) performed to their related classifiers as feature vector. In this experiment, we've used a kind of wavelet (haar) up to 3 levels of resolution \& our recognition rate was 92\%.

\subsubsection{Curvelet transform:}

By performing curvelet transform to each of input pattern, 119448 curvelet coefficients achieved. All of these coefficients in different scales \& angels performed to their related classifier as feature vector. In this case, coefficients obtained in 4 different scales with various angels, \& recognition rate became $100 \%$.

Table 1.The recognition rates with different lengths of $\mathbf{3}$ various feature vectors.

\begin{tabular}{|l|l|l|l|l|l|l|}
\hline $\begin{array}{l}\text { Number of features } \\
\text { (coefficients) }\end{array}$ & $\begin{array}{l}\text { All coefficients (FFT=16384, } \\
\text { Wavelet=16384, curvelet=119449) }\end{array}$ & 13130 & 10000 & 9000 & 8000 & 6000 \\
\hline $\begin{array}{l}\text { Recognition rate } \\
\text { using FFT }\end{array}$ & $97 \%$ & $90 \%$ & $87 \%$ & $85 \%$ & $85 \%$ & $70 \%$ \\
\hline $\begin{array}{l}\text { Recognition rate } \\
\text { using Wavelet }\end{array}$ & $92 \%$ & $89 \%$ & $89 \%$ & $87 \%$ & $90 \%$ & $85 \%$ \\
\hline $\begin{array}{l}\text { Recognition rate } \\
\text { using Curvelet }\end{array}$ & $100 \%$ & $100 \%$ & $97 \%$ & $97 \%$ & $95 \%$ & $95 \%$ \\
\hline
\end{tabular}

3.2. Reducing the dimension of feature vectors (curvelet coefficients, wavelet coefficients, Fourier coefficients)

As it is proved, the kinds of features and the sizes of feature vectors are so important in our recognizing process. On the other hand, Because of existing a large number of curvelet coefficients from backward view of vehicles if these coefficients be performed to our classifier, the speed of our recognition system will be decreased. To solve this problem, we reduced the dimension of feature vectors with using of interclass \& intraclass variance criteria. 
To be useful, a feature must exhibit small intra-class variance as well as large inter-class variance. Intra-class variance translates to variability of the feature within the target class, and a small value would indicate better representation of the feature for this class. On the other hand, inter-class variance measures the separability of different target classes using this feature, and hence, a large value is desirable [18 ].

For each input image, a set of features are extracted and placed in a vector.

This is repeated for all $N_{\text {train }}$ training data \& for all classes to form the matrix as shown in (8):

$$
X=\left[\begin{array}{c}
X(1) \\
X(2) \\
\vdots \\
X\left(N_{\text {train }}\right)
\end{array}\right]
$$

The mean feature vector, $X_{b a r}$, is extracted by averaging along each column, and each column of $\mathrm{X}$ is subtracted from $X_{b a r}$, as shown in (9):

$$
\tilde{X}=X-\left[\begin{array}{c}
X_{b a r} \\
\vdots \\
X_{b a r}
\end{array}\right]
$$

The covariance matrix is computed from $\mathrm{X}$, where each diagonal element represents the spread of each feature across the training data i.e. it gives the interclass variance of this feature, as illustrated in (10).

(10)

$$
\begin{aligned}
& \sum=X^{\prime} X=\left[\begin{array}{cccc}
\sigma_{f_{1}}^{2} & . . & . . & . . \\
\cdots & \sigma_{f_{2}}^{2} & . \cdot & . \cdot \\
\cdots & \ldots & \sigma_{f_{3}}^{2} & \ldots \\
. . & . . & . . & \sigma_{f_{N}}^{2}
\end{array}\right] \\
& \sigma_{\text {INTER }}^{2}=\left[\begin{array}{llll}
\sigma_{f_{1}}^{2} & \sigma_{f_{2}}^{2} & \ldots & \sigma_{f_{N}}^{2}
\end{array}\right]
\end{aligned}
$$

The intra-class variance of each feature can be similarly obtained by considering training data for each target class at a time. These are averaged over the number of target classes to obtain $\sigma_{\text {INTRA }}^{2}$.

Features with big $\sigma_{\text {INTER }}^{2} / \sigma_{\text {INTRA }}^{2}$ are selected in order .

The achieved recognition results of reducing dimension in 3 kinds of feature vector are proposed in Table 1. Notice, the recognition rates are proposed with various lengths of feature vectors.

According to table 1, the amount of the achieved coefficients by performing curvelet transform is extremely further than the obtained coefficients by performing Fourier \& wavelet transforms to each image. In addition the recognition rate of this system which is based on curvelet transform at the time of using all curvelet coefficients for forming the feature vector is $100 \%$.

By using dimension reduction criteria $\sigma_{\text {INTER }}^{2} / \sigma_{\text {INTRA }}^{2}$, we could list the achieved coefficients as performance of Fourier, wavelet \& curvelet transforms in order to their importance.

When we used 10 percent of the most important curvelet coefficients (13130), our recognition rate became $100 \%$ again. But, when we used 13130 Fourier and curvelet coefficients our recognition rate became in order $89 \%$ and $90 \%$.

According to table 1, 10000 important coefficients were used up and again the best recognition rate was based on curvelet (about 97\%).

We continued our work with reducing the vector dimension and eventually results showed, in spite of decreasing the vector's dimension, the recognition rate which is based on curvelet transform will propose the best recognition rate in comparison with 2 other systems which are based on fft and wavelet transfroms.

\section{Conclusion}

This paper represents the efficiency comparison of 3 different structures which are based on Fourier transform in pattern recognition. These 3 kinds of structure are based on Fast Fourier transform, Wavelet transform and Curvelet transform. The features, on the basis of the recognition is made, have been obtained using Fourier, wavelet \& curvelet transformations.

The performed numerical experiments for the recognition of the vehicles backward view have shown the superiority of the curvelet transform in comparison with 2 other ways, for extracting features from images. 
During the next works, we will use of the other classifiers to improve our system recognition rate.

\section{References}

[1] MP. Dubuisson-Jolly , S. Lakshmanan, and A. Jain. Vehicle segmentation and classification using deformable templates. IEEE Transactions Pattern Analysis and Machine Intelligence, 18(3):293-308, 1996.

[2] J. Ferryman, A . Worral, G. Sulliva, and K. Baker.A generic deformable model for vehicle recognition. In British Machine Vision Conference, pages 127-136. British Machine Vision Association, 1995.

[3] W. Wei, Q. Zhang, and M. Wang. A method of vehicle classification using models and neural networks. In IEEE Vehicular Technology Conference. IEEE, 2001.

[4] T. Crimmins, A complete set of Fourier descriptors for 2-D shapes, IEEE Trans. Syst. Man Cybern. 12 (1982) 170-179.

[5]T. Kato, Y. Ninomiya, and I. Masaki. Preceding vehicle recognition based on learning from sample images. IEEE Transactions on Intelligent Transportation Systems, 3(4):252260, 2002.

[6]A. Lai, G. Fung, and N Yung. Vehicle type classification from visual-based dimension estimation. In IEEE Intelligent Transportation Systems Conference, pages 201-206. IEEE, 2001.

[7]N. Matthews, P. An, D. Charnley, and C. Harris. Vehicle detection and recognition in greyscale imagery. Control Engineering Practice, 4(4):472-479, 1996.

[8]V.S.Petrovic and T.F.Cootes. Analysis of Features for Rigid Structure Vehicle Type Recognition.2003.

[9] L. Dlagnekov ,Video-based Car Surveillance: License Plate, Make, and Model Recognition, Thesis , in Computer Science University of California, San Diego, 2005.

[10] A. Kulkarni, Artificial Neural Networks for Image Understanding, Van Nostrand, Princeton, NJ, 1988. (1992) 436-440.

[11] P. Wunsch, A. Laine, Wavelet descriptors for multiresolution recognition of handprinted characters, Pattern Recognition 28 (1995) 1237-1249.

[12] I. Daubechies, Ten lectures on wavelets, CBMS-NSF Regional Conference Series in Applied Mathematics, Vol.61, Capital City Press, Montpelier, 1992.

[13] S. Mallat, Wavelet Tour of Signal Processing, Academic Press, New York, 1998.

[14] M.V. Wickerhauser, Lectures on wavelet packet algorithms, Department of Mathematics, Washington University, St. Louis, 1991.

[15] E. Cand Cand es and D. Donoho, "Curvelets: A surprisingly effective nonadaptive representation of objects with edges," Curves and Surface, Vanderbilt University Press, Nashville, TN sity TN, pp. 123-143, 1999.

[16] E. Cand Cand es and D. Donoho, "New tight frames of curvelets and optimal representations of objects with c2 singularities,” Tech. Rep., Department of Statistics, Stanford University, USA, November 2002.

[17] E. Cand Cand 'es and L. Demanet, "The curvelet representation of wave propagators is optimally sparse," Tech.Rep., Applied and Computational Mathematics, California Institute of Technology, USA, 2004.
[18] I. Guyon, S. Gunn, M. Nikravesh , Lofti A. Zadeh , Feature Extraction: Foundations and Applications, (Studies in Fuzziness and Soft Computing), Springer, 2006.

[19] S. Theodoridis, K. Koutroumbas, Pattern Recognition, Academic Press, New York, 1999. 